Letter

\title{
On Stiffness of Optical Self-Injection Locking
}

\author{
Anatoliy Savchenkov, Skip Williams and Andrey Matsko* * \\ OEwaves Inc., 465 North Halstead Str., Suite 140, Pasadena, CA 91107, USA; \\ anatoliy.savchenkov@oewaves.com (A.S.); Skip.Williams@oewaves.com (S.W.) \\ * Correspondence: andrey.matsko@oewaves.com; Tel.: +1-626-351-4200
}

Received: 28 September 2018; Accepted: 25 October 2018; Published: 30 October 2018

\begin{abstract}
Spectrally pure semiconductor lasers produced via self-injection locking to high quality factor monolithic optical resonators demonstrate sub-kHz instantaneous linewidth. The lasers are used in photonic sensor systems and microwave photonic oscillators benefitting from the improved spectral purity, the stability and the reduced environmental sensitivity of the lasers. The laser frequency stability is defined by both the optical resonator and the optical path of the entire system comprising the laser, the resonator, and the miscellaneous optical components. The impacts of the various destabilization factors are usually convoluted, and it is hardly possible to separate them. In this paper, we report on an experimental study of an influence of the variations of the optical path on the laser frequency stability. We have created a whispering gallery mode optical resonator having the record small thermal sensitivity, on the order of $0.1 \mathrm{ppm} /{ }^{\circ} \mathrm{C}$, and demonstrated a self-injection locked laser based on this resonator. The measured laser stability is characterized with $1 \mathrm{~s}$ Allan deviation of $10^{-12}$, limited by the thermal sensitivity of the optical path between the laser and the resonator. The thermal stabilization on the order of $10 \mu \mathrm{K}$ at $1 \mathrm{~s}$ is achieved using a standard thermo-electric element. The long term drift of the laser frequency is determined by both the fluctuations of the atmospheric pressure in the laboratory impacting the monolithic resonator and by the optical path instability.
\end{abstract}

Keywords: self injection locked laser; optical resonator; resonant Rayleigh scattering; laser frequency stability; optical whispering gallery modes; thermally compensated monolithic optical resonator

\section{Introduction}

A broad spectrum of applications of low noise lasers constantly fuels the need for their improvement and for simultaneous reduction of noise, as well as their size and environmental sensitivity. While laboratory scale lasers with extraordinarily high stability and sub-Hz linewidth have been demonstrated [1-3], there are no photonic integrated circuit (PIC) devices with sub-kHz linewidth. Whispering gallery mode resonators (WGMRs) [4] as well as microring resonators allow improvement of the laser performance without significant increase of the setup size. Stability as well as spectral purity of the microresonator-based lasers has been validated with rack-mounted support equipment and fiber laser source $[5,6]$. Semiconductor lasers integrated with high quality (Q-) factor optical resonators also have a potential for the noise reduction featuring sub-kHz integral linewidth as well as sub- $\mathrm{Hz}$ instantaneous linewidth [7-10].

The idea of stabilization of a semiconductor laser using self-injection locking to a high-Q resonator is thirty year old [11-13]. In the case of WGMRs the technique is based on resonant Rayleigh scattering. Advantages of this type of stabilization include a possibility of tight packaging of the laser and no need for the electronic locking. The light from a laser scatters back in the resonator due to its optical 
inhomogeneities [14]. The coherent back reflection increases when the frequency coincides with a mode of the resonator. This provides a fast optically filtered feedback to the laser enabling reduction of the laser linewidth. Seven orders of magnitude reduction in the laser linewidth was demonstrated with this approach [15].

Long term stability of a self-injection locked laser is limited primarily due to the environment defined fluctuations of the resonator frequency. For instance, the WGMRs usually are characterized with relative frequency thermal sensitivity on the order of $10^{-5} /{ }^{\circ} \mathrm{C}$. The ambient temperature should be stabilized a $\mu \mathrm{K}$ level to achieve less than $10 \mathrm{kHz} 1 \mathrm{~s}$ linewidth of the resonator-based laser. Such a thermal stabilization calls for undesirable high power consumption and a large package size. The problem can be resolved with a thermally compensated resonator [16-18]. Thermal compensation is achieved by using an especially designed composite resonator structure.

The goal of this work is to understand the practical limitations of the stability of self-injection locked semiconductor laser and to realize a high-stability laser using a thermally compensated resonator. In this paper we report on the development of a calcium fluoride $\left(\mathrm{CaF}_{2}\right)$ WGMR integrated with ceramic layers characterized with negative thermal expansion coefficient. We achieved two orders of magnitude thermal sensitivity reduction if compared with a bare $\mathrm{CaF}_{2} \mathrm{WGMR}$, and used this resonator for a semiconductor laser stabilization. We locked a semiconductor distributed feedback (DFB) laser to the resonator using self-injection technique and realized $1 \mathrm{~s}$ relative optical frequency stability approaching $10^{-12}$. Up to our knowledge, this is the best $1 \mathrm{~s}$ stability reported for a thermally stabilized WGMR device operating under normal atmospheric conditions. The observation means that the resonator mounted on an optical microbench was stabilized at $10^{-5}{ }^{\circ} \mathrm{C}$ at this time scale. We studied limitations of the short term stability and found that the laser frequency is extremely sensitive to the temperature fluctuations of the entire laser build. Unlike Pound-Drever-Hall locking technique [1], self-injection locking has limited stiffness. We have shown experimentally that the laser frequency can be detuned with respect to the resonator mode frequency by more than a full width at the half maximum (FWHM) of the corresponding optical mode. The detuning results from the change of the optical phase delay between the laser and the resonator. The long term frequency stability was limited by the sensitivity of the resonator to the atmospheric pressure variations. Our observation clearly shows that the entire optical path should be stabilized to create a stable self-injection locked laser.

\section{Results}

Resonant self-injection locking of a laser relies on optical feedback resulting from resonant back reflection of laser light from a resonant optical structure. Emission frequency of a laser self-injection locked by means of a high-Q resonator depends on the frequency of the resonator used for the optical feedback as well as on the optical path length between the resonator and the laser. To create a stable self-injection locked laser both these factors should be taken into account. While stability limitations of the resonators were studied in detail, the importance of the stabilization of the optical phase accumulated between the laser and the resonator was not investigated as deep. We study it in this work using a resonator with low environmental sensitivity. In this case, the resonator-related variations of the laser frequency are excluded and the instability due to variations of the optical path is revealed.

\subsection{Principles of Thermal Compensation of a WGMR}

There were multiple attempts to fabricate a monolithic resonator with reduced thermal sensitivity. For example, thermal refraction effect can be eliminated in a silica microtoroid resonator by a thin layer of polydimethylsiloxane (PDMS) evanescently interacting with the light confined in the resonator [19]. The polymer has a negative thermal refraction effect. The resonator spectrum experiences red frequency 
shift due to optical attenuation and blue frequency shift due to PDMS. The effects can cancel each other. Fluctuations of the ambient temperature still impact the frequency of the composite structure.

Thermal expansion reduction in $\mathrm{MgF}_{2}$ [20] and $\mathrm{CaF}_{2}$ [17] WGMRs was reported. The $\mathrm{MgF}_{2}$ resonators were attached to a Zerodur substrate resulting in seven times reduction of the thermal sensitivity of the resonator frequency spectrum associated with thermal expansion. Laminating a $\mathrm{CaF}_{2}$ resonator between two Zerodur layers leaded to reduction of the thermal sensitivity by a factor of three. Significantly better thermal compensation can be achieved in $\mathrm{CaF}_{2}$ WGMR integrated with ceramic layers characterized with negative thermal expansion coefficient. Resultant zero sensitivity of the WGM frequency to the ambient temperature change can be realized [18]. In this study, we created a WGMR using this recipe, locked a laser to it, and have shown that the stability of the laser is still limited. We attributed the limitation to the instability of the optical path and shown experimentally that a modification of the path allows tuning the laser emission frequency by more than a FWHM of the WGMR.

\subsection{Laser Stabilization Using a Thermally Compensated WGMR}

Creating two identical experimental setups described in Section 4 of this paper we stabilized two semiconductor lasers using compensated $\mathrm{CaF}_{2}$ WGMRs. The temperature of the WGMRs was tuned to the position when the frequency of the resonators spectra weakly depends on the ambient temperature variations. The reduction of the thermal sensitivity was two orders of magnitude. If the thermal sensitivity

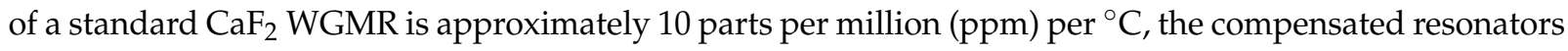
had the sensitivity of $0.1 \mathrm{ppm} /{ }^{\circ} \mathrm{C}$.

The thermal sensitivity of the standard resonator allows us to roughly evaluate the temperature variations of the resonator mode measuring the frequency variations of the laser locked to the mode. The mode frequency changes by approximately $2 \mathrm{GHz}$ per ${ }^{\circ} \mathrm{C}$. The accuracy of the measurement is given by the mode bandwidth and the lock tightness and agility. The self-injection locking mechanism is very fast. In another experiment, we have observed fast modulation of a self-injection locked laser frequency with response time approaching a $\mu$ s by modulating the frequency of the resonator using a piezo actuator. Therefore, the laser frequency is tracking the resonator mode at $1 \mathrm{~s}$ time scale. If the laser frequency moves less than $20 \mathrm{kHz}$ per $1 \mathrm{~s}$ (regularly observed in our experiments), we can say that the temperature variations of the resonator are at the level of $10 \mu^{\circ} \mathrm{C}$ or less. The accuracy of the measurement is limited because of the long term drift of the laser frequency with respect to the mode center, so the measurement shows the maximum possible temperature variations.

We created two nearly identical self-injection locked lasers involving the thermally compensated resonators. The emission of the lasers was combined in a single optical fiber and sent to a fast photodiode. The frequencies of the lasers were tuned in a way that the radio frequency (RF) signal escaping the photodiode had frequency $30 \mathrm{MHz}$. We studied stability of the signal using a frequency counter as well as a signal analyzer stabilized to an external Rubidium clocks. The signal analyzer was used to perform short term measurements, while the frequency counter was used to record the long term stability. The RF beat note of the lasers has stability defined by the least stable laser out of two.

The resonator platform was stabilized using a thermo-electric cooler. The short term thermal stability of the resonator platform was on the order $10 \mu^{\circ} \mathrm{C}$. As the result, the resonator without thermal compensation had relative frequency stability approaching $10^{-10}$ at $1 \mathrm{~s}$ integration time. The long term stability of the resonator temperature was on the order of $0.01^{\circ} \mathrm{C}$, so that the corresponding relative frequency variations were on the order of $10^{-7}$. The compensated resonator resulted in a two orders of magnitude better performance. The measurement results are illustrated in Figure 1. One can see that Allan deviation of $10^{-12}$ can be achieved at $1 \mathrm{~s}$ integration time with a compensated resonator. A frequency drift is observed at longer integration times. Up to our knowledge, this is the best $1 \mathrm{~s}$ stability reported for 
a thermally stabilized self-injection locked semiconductor laser involving a WGMR and operating under normal atmospheric conditions.

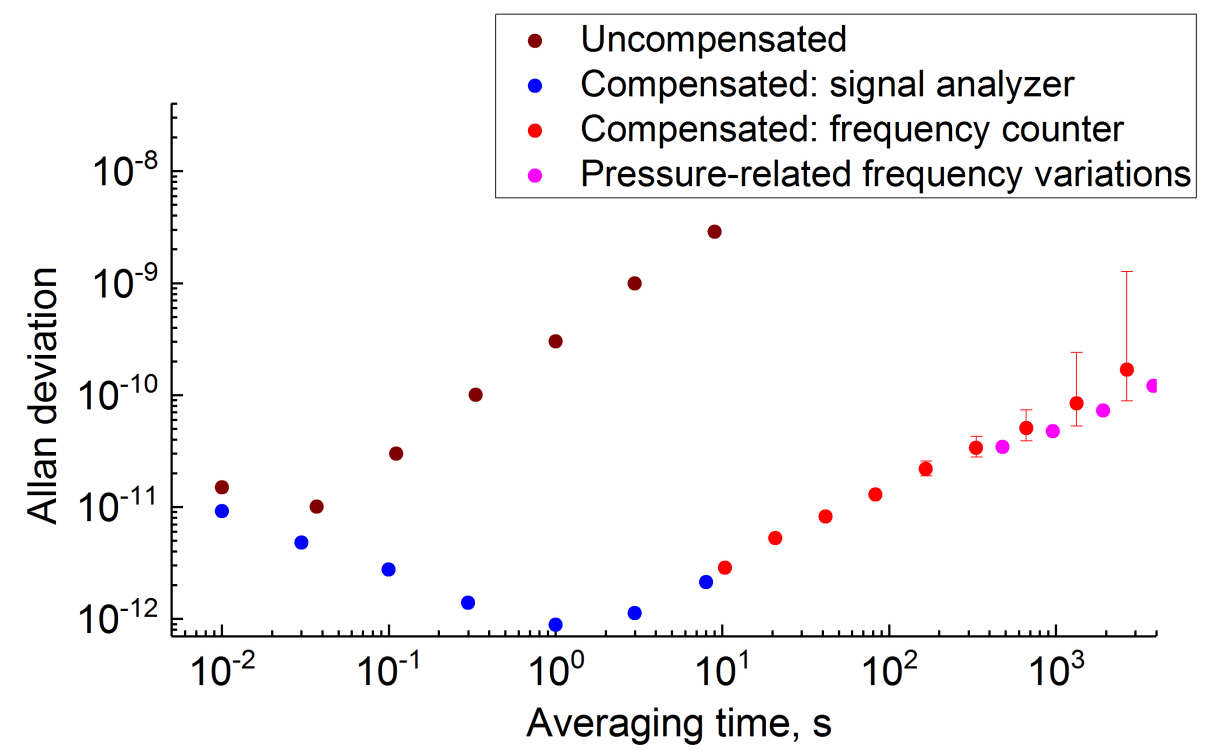

Figure 1. An illustration of stability improvement of a semiconductor DFB laser self-injection locked to a mode of a standard (brown dots) and a thermally compensated (blue and red dots) WGMR. Stability limitations due to atmospheric pressure variations acting on the resonator are shown by magenta dots. The data are indicative of approximately $10 \mu^{\circ} \mathrm{C}$ thermal stabilization of the resonator at $1 \mathrm{~s}$ integration time.

The short term Allan deviation was limited because of optical shot noise averaging due to the optical locking. Contribution of this noise was relatively large since the resonator was intentionally overloaded to $20 \mathrm{MHz}$ bandwidth. The frequency bandwidth of the resonator mode defines the short term stability of the self-injection locked laser in a similar way as the bandwidth of a laser cavity defines the linewidth of the laser (see Discussion for details). The large bandwidth was necessary to measure the variation of the laser frequency with modification of the optical path between the laser and the resonator.

Another reason for the short term frequency instability is associated with the low stiffness of the locking point of the self-injection locking system. Variations of the optical path as well as the current driving the laser result in the frequency shift of the laser emission with respect to the WGM frequency studied in this paper.

We attribute the long term frequency drift to both the variation of the atmospheric pressure and the compensated resonator temperature. The resonator was not hermetically sealed and, as was shown earlier, variations of the atmospheric pressure deform the resonator, change its refractive index because of the associated stress, and modify the refractive index of the surrounding air that can be sensed by the evanescent field of the WGMs.

The variation of the WGMR temperature also could have a sizable contribution the frequency drift. Long term temperature related relative frequency instability of the resonator on the order of $10^{-9}$ (associated with $0.01{ }^{\circ} \mathrm{C}$ long term thermal stabilization magnitude) can lead to $1 \mathrm{~s}$ instability on the order of $10^{-12}$ if the Allan deviation averaging time dependence $\tau^{1 / 2}$ is expected.

Our current study does not allow a reliable separation of the pressure and temperature associated long term frequency drifts. The resonator should be placed into an evacuated package to remove the pressure variation impact. However, even if the thermal stabilization of the resonator is improved and both these 
effects are removed, the long term stability of the self-injection locked laser, similarly to the short-term stability, still can be compromised due to low tightness of the self-injection locking. As shown below, the entire platform, not only the WGMR, has to be thermally stabilized to improve the stabilization quality. The thermal instability of the platform also can explain the observed $10^{-12}$ relative frequency stability.

\subsection{Tightness of Self-Injection Lock}

Self-injection locking is a frequency pulling phenomenon. The emission of the laser is determined by the frequency of the laser cavity, frequency of the WGMR, and phase delay associated with the optical path. By creating a stable WGMR we can reduce the impact of the WGMR on the frequency of the laser and figure out the impact of the other two factors on the laser frequency.

\subsubsection{Laser Current Change}

Change of the laser current modifies both the frequency and the power of the laser emission. In the self-injection locked system it changes partial frequency of the laser. By "partial frequency" here we understand the frequency of the laser emission observed in a free running, not self-injection locked, laser. The actual laser frequency changes much slower because of the self-injection locking. We demonstrated this effect experimentally (Figure 2). We measured frequency tuning of the laser using a frequency etalon as a discriminator, Figure 2a. The self-injection locking range was monitored using the LI-curve (dependence of the power of the laser on the laser current), Figure $2 \mathrm{~b}$. The classical curve for the self-injection locked laser emission frequency versus partial frequency of the laser chip was created using the data, Figure 2c.
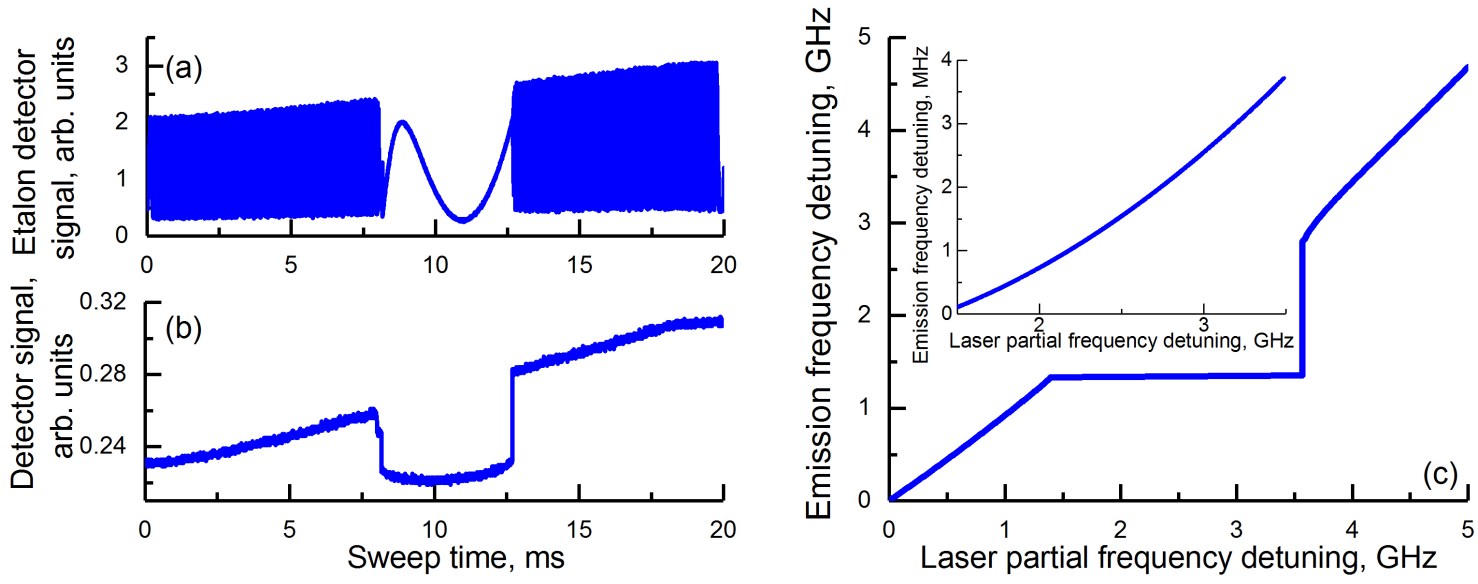

Figure 2. Measured dependence of the laser frequency (a), and power (b), on the laser current. The frequency is measured by sending laser light through an etalon while the power dependence is measured using a photodiode while laser current is scanning; (c) Dependence of the laser emission frequency on the partial frequency of the free running laser. Inset shows tuning of the self-injection locked laser within the locking range. The data are inferred from the measurement results shown in panel (a).

We modulated the laser current using a saw tooth signal. The center of the modulation was selected in a way that the laser emission hits a WGM and the laser locks to it. The locking can be visualized with a slow photodiode. The photocurrent linearly increases with the driving current increase. At this point the light from the laser has relatively large linewidth and it does not enter the resonator. The photocurrent drops suddenly when the laser locks to the mode since a part of the light is reflected (scattered) back to the laser. This process is illustrated in Figure $2 b$. 
The power change is associated with the frequency change. To characterize the laser locking frequency we used a Fabry-Perot etalon with $5 \mathrm{MHz}$ free spectral range (FSR) followed by another slow photodiode. When the laser frequency is scanned through the etalon, the photocurrent is modulated with a fast rate associated with the frequency change with respect to the etalon modes. Locking of the laser results in reduction of the frequency tuning so the modulation frequency suddenly decreases. When the laser unlocks the frequency start changing again. In the vicinity of the locking point the frequency change can be too fast to be measured. The process is illustrated in Figure 2a.

Knowledge of the etalon FSR allows us to find dependence of the laser frequency on the current magnitude and the current ramp speed and calibrate the measurement results in the frequency units. The result of the calibration of the measurement data with respect to the laser partial frequency is shown in Figure 2c. One can see that the laser frequency tuning reduces by 1000 times when the laser is locked to the mode of the resonator. The overall tuning range is approximately $20 \%$ of the resonator FWMH.

The locking range of the laser exceeds the bandwidth of the mode significantly. Ultimately, the locking range is limited by the bandwidth of the laser chip gain and the laser emission frequency can be tuned continuously by tuning the WGM frequency within the bandwidth of the laser chip gain. The observed locking range value is much narrower in our experiment since we do not adjust the other parameters of the system during the measurement. To achieve a much broader tuning one needs to modify the phase delay between the laser and the resonator while changing the resonator frequency.

The measurement shows that if the frequency tuning of the free running laser is $0.42 \mathrm{GHz} / \mathrm{mA}$, the tuning of the self-injection locked laser is $0.4 \mathrm{MHz} / \mathrm{mA}$. To achieve relative stability of the laser at the level of $10^{-12}$, the associated driving current variation should be $0.5 \mu \mathrm{A}$ at $1 \mathrm{~s}$. This is a rather small value for a commercial current driver, however we apparently achieved it in our experiment.

The locking can be made tighter if the frequency bandwidth of the WGM is reduced. A calcium fluoride WGMR can have bandwidth of $10 \mathrm{kHz}$ [21]. The laser locked to the narrow mode should be tunable by a few $\mathrm{kHz}$ while locked. However, measurement of the frequency shift is not simple at this level. We had to overload the resonator to measure the effect using a standard etalon. That is why the Q-factor of the resonator used in the current experiment was relatively low.

\subsubsection{Optical Path Change}

To understand how the optical path length change impacts the frequency shift of the self-injection locked laser we introduced a tunable phase delay in between of the laser and the resonator, as described in Section 4 of the paper. By changing the optical path length we were able to tune the frequency of the laser through injection locked region. This tuning emulates the otherwise uncontrollable optical path variations in self-injection locked lasers.

The experiment was performed as follows. Keeping the temperature of the phase section as well as the laser current constant we tuned the temperature of the resonator to observe the locking. The locking was observed when the partial temperature of the laser coincided with the resonator mode frequency and the phase delay between the laser and the resonator was optimal. Then we fixed the temperature of the resonator and ramped the temperature of the phase shifter. The experiment shown that there is a limited range of the phase shifter temperatures for which self-injection locking was conserved.

Unlike the case of the laser frequency tuning described in the previous section, the phase shifter did not influence the laser frequency and power when the laser was not locked to the resonator mode, Figure 3. When the locking occurred, the phase shifter impacted the emission frequency of the system. Measuring the frequency shift using the Fabry-Perot etalon we observed that the absolute value of the emission frequency was shifted by approximately $80 \mathrm{MHz}$, or more than four FWHMa of the WGM. This is a very large number. 
In the worst case scenario the shift rate was $10 \mathrm{MHz} /{ }^{\circ} \mathrm{C}$. To estimate it we have noticed that the optical frequency shifts $80 \mathrm{MHz}$ per approximately $\lambda / 2$ optical path change. The wavelength is $\lambda=1.55 \mu \mathrm{m}$, the optical path length is $L \approx 1 \mathrm{~cm}$. Assuming thermal expansion of $10^{-5} /{ }^{\circ} \mathrm{C}$, we find that the temperature change needed for the change of the path by $\lambda / 2$ is $8^{\circ} \mathrm{C}$. This results in the frequency rate change with temperature variations of the build.

The observation means that the temperature of the entire system should be stabilized to approximately $20 \mu^{\circ} \mathrm{C}$ level to achieve relative frequency stability of $10^{-12}$. This level of thermal stabilization of the entire platform is hard to maintain for a long time. On the other hand, it is clear from the measurement that there is a certain length of the optical path that corresponds to the turning point at which the variations of the setup temperature do not impact the laser frequency. In addition, improving Q-factor of the resonator by several orders of magnitude results in softening the requirements to the temperature stabilization of the optical path.


Figure 3. Measured dependence of the laser frequency (a), and power (b), on the change of the optical path from the laser to the resonator. The optical path change shown in units of time corresponding to the temperature rump of the phase section. The optical path is varied by scanning the temperature of the phase section between the laser and the resonator. The optical frequency is measured by sending laser light through an etalon while the power dependence is measured using a photodiode; (c) Variation of the self-injection locked laser emission frequency while optical path between the laser and the resonator is changing in time. The data are inferred from the measurement shown on panel (a). The laser frequency is undefined outside of the locking range since the change of the optical path does not impact the frequency of the free running (unlocked) laser.

In our experiment intended for achieving the highest possible stabilization of the laser we selected the phase in such a way that the turning point was reached. The system did not have an active stabilization of the optical path and due to thermal drift the moved from the desirable point approximately in an hour. This feature limited the stability measurements (Figure 1) by approximately $1000 \mathrm{~s}$.

Interestingly, the nonlinear dependence of the frequency shift of the self-injection locked laser on the phase delay between the WGM resonator and the laser chip complicate the stabilization of the resonator frequency using external optical clock transition. The temperature of the resonator can vary in a frequency range determined by the stiffness of the lock even if we stabilize the laser to the atomic cell. To avoid this discrepancy, one has to stabilize actively the optical path between the laser and the resonator and also use a high-Q resonator. 


\section{Discussion}

Self-injection locking of a semiconductor laser to a high-Q resonator results in a sizable reduction of the laser frequency noise and linewidth [11-13]. While a significant amount of research devoted to the study of the process was performed, not much of it was focused at self-injection locking using WGMRs. A few experiments on self-injection locking of lasers to WGMRs have been reported [7-10]. The theoretical research was even more scarce [22,23]. An analytical expression for the spectral bandwidth of the locking was derived in [23]. The frequency tuning of the self-injection locked laser system achieved by changing the WGM frequency was also discussed in that paper. Out current experimental research is explained well by the theory.

The expression for the locking range $\Delta v$

$$
\frac{\Delta v}{v}=\sqrt{1+\alpha_{g}^{2}} \frac{\Gamma_{m}}{Q_{d}}
$$

presented in [23], describes relatively well our observation. In our case the carrier frequency is $v=2 \times 10^{14} \mathrm{~Hz}$, alpha (line enhancement) factor is $\alpha_{g} \simeq 2.5$, amplitude reflection back into the laser diode $\Gamma_{m} \simeq 0.1$, quality factor of the laser cavity $Q_{d} \simeq 10^{4}$. The formula results in $\Delta v \simeq 5.4 \mathrm{GHz}$, which is close to locking range of $2.5 \mathrm{GHz}$ illustrated in Figure 2.

The theory [23] also predicts the periodic behavior of the self-injection locking with respect of the optical phase delay. We have increased the tuning range to cover the optical path change by a few optical wavelength and observed periodic behavior of the self-injection locking Figure 4, as expected. It is possible to see that the laser locks for $3 / 4$ of the period and the power changes rather significantly. This measurement clearly illustrates a way of change the frequency detuning between the laser emission and the WGM.

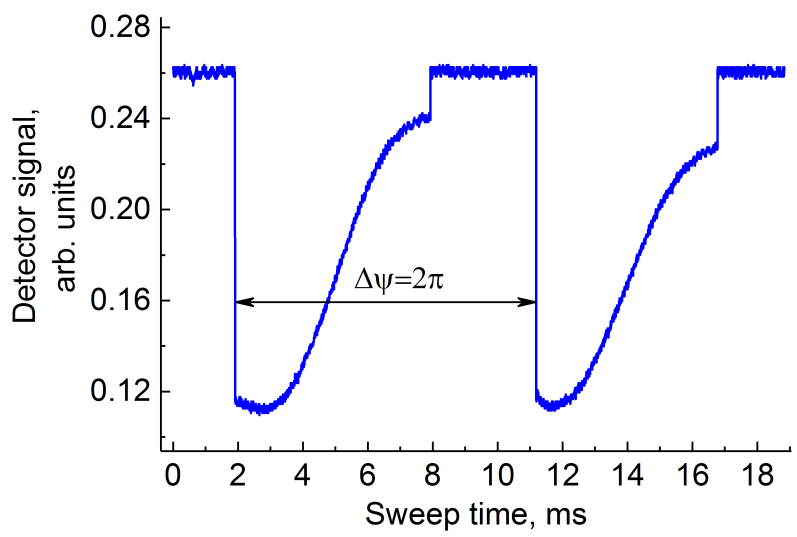

Figure 4. Measured dependence of the laser power on the change of the optical path from the laser to the resonator for a broader optical phase tuning range. The period of the curve corresponds to the optical phase change $\Delta \phi=2 \pi$. The locking is conserved for $\Delta \phi \simeq 3 \pi / 2$.

To understand qualitatively the impact of the optical path length on the frequency of the self-injection locked laser we can involve an analogy between the laser and a multi-cavity oscillator. The frequency of such an oscillator is given by the normal modes of the multi-cavity system and, generally, each cavity impacts the frequency. The self-injection locked laser involves three contours represented by the laser cavity, the WGM resonator, and the optical delay line between the laser and the WGM resonator. The lasing frequency is determined by the normal mode that is impacted by all the contours. The short-term stability of the system is mostly given by the partial frequency of the WGM. However, the long-term stability is 
impacted by the laser cavity and the optical path between the laser and the resonator. That is why it is so important to stabilize not only the resonator but also the optical path length to achieve high stability of the self-injection locked laser system.

\section{Materials and Methods}

The experimental setup is shown in Figure 5. Light from a semiconductor distributed feedback (DFB) laser chip attached to a submount is focused and send through a tunable phase shifter (an optical window mounted on a heater) an an evanescent prism coupler to a WGMR. All the parts are bonded to the substrate, so any variations of the optical path are suppressed to a possible technical minimum.

The laser chips used in the experiment are the standard C-band Dense Wavelength Division Multiplexing(DWDM) Distributed Feedback (DFB) lasers. The laser driving current is $600 \mathrm{~mA}$ and the emission power is $40 \mathrm{~mW}$. Thermal sensitivity of the laser wavelength is $0.1 \mathrm{~nm} /{ }^{\circ} \mathrm{C}$. Linewidth of the laser emission is approximately $1 \mathrm{MHz}$.

Two nearly identical setups are created to perform the stability measurements. The output of the setups are sent to a fast photodiode generating an RF signal corresponding to the laser beat note frequency. The frequency stability is studied using an RF frequency counter and a signal analyzer.

The highest finesse of solid state optical resonators was achieved with $\mathrm{CaF}_{2}$ WGMRs [21]. The resonators have large thermal expansion $\left(19 \mathrm{ppm} /{ }^{\circ} \mathrm{C}\right)$ and thermo-refraction $\left(-8.2 \mathrm{ppm} /{ }^{\circ} \mathrm{C}\right)$ at $1550 \mathrm{~nm}$ resulting in the high sensitivity to the ambient temperature variations. The thermo-refractive coefficient of the mounted resonator is slightly different because of the temperature associated change of the refractive index of the resonator due to the stress. The stress as well as stress propagation depends on the mounting geometry. We have found that using $\mathrm{CaF}_{2}$ resonator laminated with Zerodur (Clearceram- $\mathrm{Z}$ HS) layers one is able to reduce the thermal sensitivity of the resonator to nearly zero. This type of the resonator is used in the experiment described in this paper. The temperature stabilization factor approaches two orders of magnitude.



Figure 5. Schematic of the experimental setup.

Picture of the $\mathrm{CaF}_{2}$ WGMR is shown in the inset of Figure 5. The resonator is designed in a way that stress does not propagate significantly the WGM volume. The resonator has $0.1 \mathrm{~cm}$ in diameter and $50 \mu \mathrm{m}$ 
thickness of the $\mathrm{CaF}_{2}$ layer. Optical WGM is localized in this layer. The compensated layers have $0.09 \mathrm{~cm}$ in diameter and $58 \mu \mathrm{m}$ thickness. The fabricated resonator has intrinsic Q-factor exceeding a $10^{9}$ at $1.5 \mu \mathrm{m}$ wavelength. The resonator is overloaded to $20 \mathrm{MHz}$ FWHM. The low Q-factor is selected to be able to measure the change of the laser frequency with the optical phase change and to avoid frequency shifts associated with the power build up in the resonator mode. The optical backscattering is strong enough to support the self-injection locking of the laser to the resonator.

\section{Conclusions}

In this paper, we demonstrate a self-injection locked laser characterized with a passive stability of $10^{-12}$ at $1 \mathrm{~s}$ integration time. The stability is limited because of the low stiffness of the self-injection locking mechanism and due to the temperature as well as the atmospheric pressure variations. Integration of the lasers in evacuated thermally stabilized enclosures, introduction of the active stabilization of the optical path and usage of high-Q thermally compensated resonators are promising for further improvement of the laser stability.

Author Contributions: A.S. and A.M. conceived the experiment; A.S. designed and performed the experiment; A.M. analyzed the data; A.M. and S.W. wrote the paper.

Funding: The project was supported by internal R\&D funding.

Acknowledgments: Andrey Matsko acknowledges useful and helpful discussions with Danny Fung.

Conflicts of Interest: The authors declare no conflict of interest.

\section{References}

1. Drever, R.W.P.; Hall, J.L.; Kowalski, F.V.; Hough, J.; Ford, G.M.; Munley, A.J.; Ward, H. Laser phase and frequency stabilization using an optical resonator. Appl. Phys. B 1983, 31, 97-105. [CrossRef]

2. Young, B.C.; Cruz, F.C.; Itano, W.M.; Bergquist, J.C. Visible lasers with subhertz linewidths. Phys. Rev. Lett. 1999, 82, 3799-3802. [CrossRef]

3. Kessler, T.; Hagemann, C.; Grebing, C.; Legero, T.; Sterr, U.; Riehle, F.; Martin, M.J.; Chen, L.; Ye, J. A sub-40-mHz-linewidth laser based on a silicon single-crystal optical cavity. Nat. Photonics 2012, 6, 687-692. [CrossRef]

4. Matsko, A.B.; Ilchenko, V.S. Optical resonators with whispering gallery modes I: Basics. IEEE J. Sel. Top. Quantum Electr. 2006, 12, 3-14. [CrossRef]

5. Lee, H.; Suh, M.-G.; Chen, T.; Li, J.; Diddams, S.A.; Vahala, K.J. Spiral resonators for on-chip laser frequency stabilization. Nat. Commun. 2013, 4, 2468. [CrossRef] [PubMed]

6. Li, J.; Lee, H.; Vahala, K.J. Microwave synthesizer using an on-chip Brillouin oscillator. Nat. Commun. 2013, 4, 2097. [CrossRef] [PubMed]

7. Vassiliev, V.V.; Velichansky, V.L.; Ilchenko, V.S.; Gorodetsky, M.L.; Hollberg, L.; Yarovitsky, A.V. Narrow-line-width diode laser with a high-Q microsphere resonator. Opt. Commun. 1998, 158, 305-312. [CrossRef]

8. Vassiliev, V.V.; Ilina, S.M.; Velichansky, V.L. Diode laser coupled to a high-Q microcavity via a GRIN lens. Appl. Phys. B 2003, 76, 521-523. [CrossRef]

9. Kieu, K.; Mansuripur, M. Fiber laser using a microsphere resonator as a feedback element. Opt. Lett. 2007, 32, 244-246. [CrossRef] [PubMed]

10. Sprenger, B.; Schwefel, H.G.L.; Wang, L.J. Whispering-gallery-mode-resonator-stabilized narrow-linewidth fiber loop laser. Opt. Lett. 2009, 34, 3370-3372. [CrossRef] [PubMed]

11. Dahmani, B.; Hollberg, L.; Drullinger, R. Frequency stabilization of semiconductor lasers by resonant optical feedback. Opt. Lett. 1987, 12, 876-878. [CrossRef] [PubMed]

12. Hollberg, L.; Ohtsu, M. Modulatable narrow-linewidth semiconductor lasers. Appl. Phys. Lett. 1988, 53, 944-946. [CrossRef] 
13. Hemmerich, A.; Zimmermann, C.; Han̈sch, T.W. Compact source of coherent blue light. Appl. Opt. 1994, 33, 988-991. [CrossRef] [PubMed]

14. Gorodetsky, M.L.; Pryamikov, A.D.; Ilchenko, V.S. Rayleigh scattering in high-Q microspheres. J. Opt. Soc. Am. B 2000, 17, 1051-1057. [CrossRef]

15. Liang, W.; Ilchenko, V.S.; Eliyahu, D.; Savchenkov, A.A.; Matsko, A.B.; Seidel, D.; Maleki, L. Ultralow noise miniature external cavity semiconductor laser. Nat. Commun. 2015, 6, 7371. [CrossRef] [PubMed]

16. Savchenkov, A.A.; Matsko, A.B.; Ilchenko, V.S.; Yu, N.; Maleki, L. Whispering-gallery-mode resonators as frequency references. II. Stabilization. J. Opt. Soc. Am. B 2007, 24, 2988-2997. [CrossRef]

17. Baumgartel, L. Whispering Gallery Mode Resonators for Frequency Metrology Applications. Ph.D. Thesis, University of Southern California, Los Angeles, CA, USA, December 2013.

18. Savchenkov, A.; Matsko, A. Calcium fluoride whispering gallery mode optical resonator with reduced thermal sensitivity. J. Opt. 2018, 20, 035801. [CrossRef]

19. He, L.; Xiao, Y.F.; Dong, C.; Zhu, J.; Gaddam, V.; Yang, L. Compensation of thermal refraction effect in high-Q toroidal microresonator by polydimethylsiloxane coating. Appl. Phys. Lett. 2008, 93, 201102. [CrossRef]

20. Lim, J.; Savchenkov, A.A.; Dale, E.; Liang, W.; Eliyahu, D.; Ilchenko, V.; Matsko, A.B.; Maleki, L.; Wong, C.W. Chasing the thermodynamical noise limit in whispering-gallery-mode resonators for ultrastable laser frequency stabilization. Nat. Commun. 2017, 8, 8. [CrossRef] [PubMed]

21. Savchenkov, A.A.; Matsko, A.B.; Ilchenko, V.S.; Maleki, L. Optical resonators with ten million finesse. Opt. Express 2007, 15, 6768-6773. [CrossRef] [PubMed]

22. Oraevsky, A.N.; Yarovitsky, A.V.; Velichansky, V.L. Frequency stabilisation of a diode laser by a whispering gallery mode. Quantum Electron. 2001, 31, 897-903. [CrossRef]

23. Kondratiev, N.M.; Lobanov, V.E.; Cherenkov, A.V.; Voloshin, A.S.; Pavlov, N.G.; Koptyaev, S.; Gorodetsky, M.L. Self-injection locking of a laser diode to a high-Q WGM microresonator. Opt. Express 2017, 25, 28167-28178. [CrossRef]

(C) 2018 by the authors. Licensee MDPI, Basel, Switzerland. This article is an open access article distributed under the terms and conditions of the Creative Commons Attribution (CC BY) license (http:/ / creativecommons.org/licenses/by/4.0/). 